\title{
A Facile Strategy to Fabricate Silver-Functionalized Superhydrophobic Cotton Fabrics with Long-Term Antibacterial Properties
}

\section{Zhanpeng Ye}

Tianjin University

Hui Rong

Tianjin University

Shuangyang Li

Tianjin University

Qing Ma

Tianjin University

Liandong Deng

Tianjin University

Jianhua Zhang ( $\boldsymbol{\nabla}$ jhzhang@tju.edu.cn )

Tianjin University

Anjie Dong

Tianjin University School of Chemical Engineering And Technology

\section{Research Article}

Keywords: silver nanoparticle, mesoporous silica nanoparticles, antibacterial fabrics, non-wetting, longterm

Posted Date: August 13th, 2021

DOI: https://doi.org/10.21203/rs.3.rs-696268/v1

License: (c) (1) This work is licensed under a Creative Commons Attribution 4.0 International License. Read Full License

Version of Record: A version of this preprint was published at Cellulose on November 25th, 2021. See the published version at https://doi.org/10.1007/s10570-021-04289-5. 


\section{Abstract}

Silver-functionalized textiles, have attracted considerable attention as the most common protective medical materials. However, their poor antibacterial durability and relatively tedious preparation process limit their applications. In this study, a kind of multifunctional cotton fabric was prepared through simultaneous dip-coating of silver-silica hybrid nanoparticles (Ag-MSNs) and fluorinated MSNs (F-MSNs), leading to excellent antifouling due to superhydrophobicity and long-term antibacterial properties from the sustained release of $\mathrm{Ag}$ ions $\left(\mathrm{Ag}^{+}\right)$. Detailed studies were performed to evaluate their structure and protective performance, especially the long-term antibacterial properties of the obtained fabrics through the inhibition zone experiment for $25 \mathrm{~d}$. The as-prepared fabrics showed good non-wetting properties and sustained antibacterial activity against Escherichia coli (E. coli) and Staphylococcus aureus (S. aureus). Further washing and abrasion experimentations demonstrated that the as-prepared fabrics had stable hydrophobicity and antibacterial properties, much more reliable than commercially derived antibacterial gauze. Considering the readily available inexpensive materials and simple preparation process as well as long-term and efficient sterilization effect, the strategy provided herein opens a new and facile way to fabricate multifunctional fabrics with robust hydrophobic and antibacterial activity for the future medical materials.

\section{Introduction}

Cotton fabrics have a wide range of application scenarios in civil and military applications as their good air permeability, wearing comfort, and other special protective properties (Xu et al. 2017; Wu et al. 2016). Although cotton fabrics provide a lot of unique protective properties, the porous structure and hydrophilic properties of the fabrics make them easy to absorb numbers of bacteria or fungi on the surface, further resulting in adverse effects on the properties of the fabrics (Elshaarawy et al. 2019). Especially, when cotton fabrics, infected by microorganisms, are in close contact with the wound, the microorganisms are very easy to grow and multiply in large numbers, and eventually leading to serious wound infection (Zhang et al. 2015; Li et al. 2011). Recently, tremendous efforts have been dedicated to the development of functionalized fabrics with antibacterial activity (Xu et al. 2017; Liu et al. 2012; Holt et al. 2018). Surface modification of cotton fabrics with significantly antibacterial substances is one of the most acceptable methods. A variety of metal ions (Rizzello et al. 2014), polymers (Lin et al. 2018; Fan et al. 2018), and nanomaterials (Fu et al. 2017) with antibacterial properties have been widely used to design antibacterial cotton fabrics. Among them, silver ions are the most attractive ones which belong to the release-killing antibacterial materials for their broad-spectrum bactericidal properties on both Gramnegative and Gram-positive bacteria (Jin et al. 2018). As the most common antibacterial fabrics, silverfunctionalized fabrics are widely used in medical care and special military equipment due to their excellent sterilization and applicability (Ouadil et al. 2019; Syafiuddin et al. 2019; Krishnan et al. 2020).

Currently, there are two main methods for preparing silver-functionalized fabrics. One is to add silver nanoparticles (Ag NPs) in the spinning process to form antibacterial fibers and then Ag NPs-doped fibers are weaved by the fibril method (Yu et al. 2021). Ag NPs can be well and durably introduced by the fibril 
method. However, this method is relatively complicated. Moreover, the prepared nanofibers have some gaps in mechanical and tensile strength compared with ordinary fibers. The flexibility and comfort of the functionalized fabrics would also deteriorate (Zhang et al. 2016). The other is to form Ag NPs on the surface of the fabrics in situ or to coat the prepared Ag NPs on the fabrics by finishing methods such as coating, sputtering, and printing (Krishnan et al. 2020; Xu et al. 2019; Chauhan et al. 2019; Ou et al. 2019). Compared with the fibril method, the finishing method is simpler in operation, but the Ag NPs are easily fall off or suffer from uncontrolled release (Qiu et al. 2020). As a result, the silver-functionalized fabrics prepared by this method have poor durability and low antibacterial stability. In addition, it was worth pointing out that the aggregation of Ag NPs is often unavoidable for the preparation of silverfunctionalized fabrics leading to difficulty for large-scale industrial production (Yu et al. 2021). Consequently, it is highly worthy to explore a facile and efficient approach to introduce suitable Ag NPs onto the fabrics to produce antibacterial fibers with the durable coalition and controlled distribution of $\mathrm{Ag}$ NPs.

For current silver-functionalized fabrics, their applications still suffer from antibacterial durability. The antibacterial properties of the silver-functionalized fabrics will gradually decrease and eventually disappear, with the continuous consumption of $\mathrm{Ag}^{+}$during daily use, leading to the infection by microorganisms again (Kim et al. 2018). Therefore, it is particularly important to achieve the long-term antibacterial properties of silver-functionalized fabrics. To achieve the long-term high-efficiency antibacterial effect, it is necessary to load more Ag NPs or reduce the release rate of $\mathrm{Ag}^{+}$without affecting the antibacterial effect. Unfortunately, the loaded amount of Ag NPs was limited by the aggregation of Ag NPs and the deteriorated effect on the flexibility, breathability, and comfort of fabrics ( $\mathrm{Li}$ et al. 2017). Slowing down the release rate of $\mathrm{Ag}^{+}$can effectively extend their antibacterial action time when the fabrics are modified with certain silver-loaded functionalized nanoparticles. However, the antibacterial ability of the silver-functionalized fabrics will be greatly reduced (Jin et al. 2018). There is a big challenge to resolve the tradeoff between effective antibacterial efficacy by sufficient release of $\mathrm{Ag}^{+}$and long-term antibacterial properties by slow consumption of $\mathrm{Ag}^{+}$. Recently, the superhydrophobic modifications by introducing low surface energy substances and surface micro-nano structures have attracted great attention as the main antifouling strategies for the fabrication of antibacterial surfaces. Superhydrophobic surfaces are defined as contact angles (CAs) larger than $150^{\circ}$, and sliding angles (SAs) less than $10^{\circ}$ (Wang et al. 2020), which possess low adhesion to bacteria (Li et al.2017). In this case, the amounts of bacteria attached per unit area of the superhydrophobic fabrics can be significantly reduced. As a result, the small amounts of adhering bacteria can be easily killed by a low concentration of $\mathrm{Ag}^{+}$. That is to say, even if the release of $\mathrm{Ag}^{+}$from silver-functionalized superhydrophobic fabrics is slow, the outstanding bactericidal effect can be achieved.

Inspired by superhydrophobic antibacterial surface, we propose an effective and facile strategy to fabricate cotton fabrics with outstanding antibacterial performance and superhydrophobic properties through simultaneous dip-coating of silver-silica hybrid nanoparticles (Ag-MSNs) and fluorinated MSNs (F-MSNs), as shown in Scheme. The Ag-MSNs with good dispersibility and F-MSNs as 
low surface energy materials were firmly glued on the cotton fabrics by polydimethylsiloxane (PDMS). Ag-MSNs, formed by the composition of Ag NPs and MSNs, could be firmly bonded to the surface of the fabrics by PDMS owing to the high specific surface areas of MSNs, thereby leading to a high bonding strength and durable coating of Ag NPs (Nie et al. 2018). The low surface energy and nanostructures of F-MSNs, coated to the surface of the fabrics by PDMS, resulted in superhydrophobic and antifouling properties for coated fabrics. PDMS acted as a binder in the one-step dip-coating process that could not only firmly anchor the Ag-MSNs and F-MSNs but also slow down the release rate of $\mathrm{Ag}^{+}$. As a result, the as-prepared fabrics had both stable superhydrophobicity $\left(C A=151 \pm 1^{\circ}\right)$ for antifouling performance and long-term antibacterial properties (zone of inhibition for $25 \mathrm{~d}$ ) for reliable protection.

\section{Experimental Section}

\subsection{Materials.}

Silver nitrate $\left(\mathrm{AgNO}_{3}, 0.0242 \mathrm{~mol} / \mathrm{L}\right)$, Tetraethyl orthosilicate (TEOS, $\geq 99 \%$ ), trimethoxy

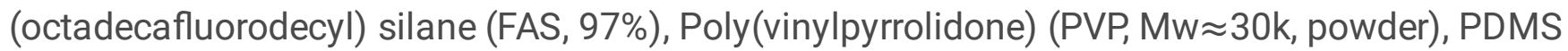
(prepolymer named as PDMS-A and the thermal curing agent named as PDMS-B) was purchased from Dow Corning Corp. Diethanolamine (DEA囚 $\geq 99.5 \%$ ), methanol, ethanol, tetrahydrofuran (THF, $\geq 99.5 \%$ ), hexadecyltrimethylammonium chloride (CTAC, 25 wt.\%), and hydrochloric acid (37\%, analytical grade), were purchased from Sigma-Aldrich. Gram-positive bacteria Staphylococcus aureus (S. aureus, ATCC 6538) and Gram-negative bacteria Escherichia coli (E. coli, ATCC 25922) were incubated at $37^{\circ} \mathrm{C}$ for $24 \mathrm{~h}$ before use. The cotton fabrics $\left(200 \mathrm{~g} / \mathrm{m}^{2}\right)$ were purchased from a local store. All these above-mentioned reagents were used as received.

\subsection{Preparation of two nanoparticles (MSNs and F-MSNs)}

MSNs were synthesized with the use of the Stöber method according to the previous paper (Wu et al. 2019). Firstly, the solution of ethanol $(9 \mathrm{~mL})$ and water $(64 \mathrm{~mL})$ with $0.10 \mathrm{~g}$ of CTAC was magnetically stirred for $3 \mathrm{~min}$. After that, the DEA (0.02 g) was added to control the $\mathrm{pH}$ of the solution. And then, $7 \mathrm{~mL}$ of TEOS was added dropwise while the above solution was still stirred. The reaction mixture gradually became milky white due to the formation of silica particles at $60^{\circ} \mathrm{C}$. After $3 \mathrm{~h}$, the silica particles were obtained by centrifugation at $8500 \mathrm{rpm}$ for $15 \mathrm{~min}$. To remove the unreacted materials, the above silica particles were washed three times with ethanol and water respectively. In addition, F-MSNs were synthesized by the same process except that $1.0 \mathrm{~g}$ of FAS was also slowly added to the solution. To remove the CTAC which was the template in the above silica particles, two kinds of particles were all dispersed in $500 \mathrm{~mL}$ of the methanol solution with hydrochloric acid $(30 \mathrm{~mL})$, and the solution was refluxed for $24 \mathrm{~h}$ at $40^{\circ} \mathrm{C}$, respectively. To ensure that there was no residual hydrochloric acid, the particles after centrifuged were washed with distilled water and ethanol three times, respectively. Finally, two kinds of mesoporous nanoparticles were obtained after drying in a vacuum oven for $24 \mathrm{~h}$.

\subsection{Preparation of Ag-MSNs}


Ag-MSNs were synthesized according to a previous report with appropriate modifications (Nie et al. 2018). Firstly, $1.0 \mathrm{~g}$ of MSNs was ultrasonically dispersed in $100 \mathrm{~mL}$ of ethanol, and then the mixture was stirred for $7 \mathrm{~h}$ at $45^{\circ} \mathrm{C}$. Whereafter, $87.5 \mathrm{mg}$ of $\mathrm{AgNO}_{3}$ was slowly added into the above mixture under vigorous stirring and refluxed in the dark. And then, $2 \mathrm{~mL}$ ethanol mixed with $0.02 \mathrm{~g} \mathrm{NaBH}_{4}$ was added dropwise and stirred for $3 \mathrm{~h}$. Finally, Ag-MSNs were separated by using a centrifuge and washed with ethanol and water to remove the superfluous $\mathrm{Ag}^{+}$if any, and the precipitates were dried at $25^{\circ} \mathrm{C}$.

\subsection{Preparation of functionalized fabrics}

To obtain functionalized fabrics, the $0.15 \mathrm{~g}$ of Ag-MSNs and $0.12 \mathrm{~g} \mathrm{F-MSNs}$ were immersed into $30 \mathrm{~mL}$ THF containing $0.15 \mathrm{~g}$ of PDMS -A and $0.015 \mathrm{~g}$ PDMS-B by ultrasonic for $45 \mathrm{~min}$. After that, clear cotton fabrics $(4 \mathrm{~cm} \times 10 \mathrm{~cm})$ were put into the above solution under vortex shocking for $15 \mathrm{~min}$. Finally, the fabrics were placed into the beaker and cured at $70^{\circ} \mathrm{C}$ for $3 \mathrm{~h}$.

\subsection{Characterization of F-MSNs, Ag-MSNs and functionalized fabrics}

Surface microstructures and morphology of F-MSNs, Ag-MSNs, and functionalized fabrics were obtained by TEM and SEM, and element mapping images were obtained from an energy-dispersive spectroscope which was attached to the SEM. The chemical functional groups of the nanoparticles were analyzed by a Fourier transform infrared spectroscopy (FTIR, Thermo Fisher Scientific Inc.) which covers a 250-4000 $\mathrm{cm}^{-1}$ infrared range with a resolution of $2 \mathrm{~cm}^{-1}$. A Kruss Easy Drop goniometer (Kruss Germany) was used to measure the water contact angles (CAs) and sliding angles (SAs) of each sample. Distilled water droplets with a volume of $8 \mu \mathrm{L}$ were deposited on the samples at $25^{\circ} \mathrm{C}$. The functionalized fabrics were glued to the glass plate by double-sided tape before measured to ensure the accuracy of CAs and SAs, and average CAs and SAs had been measured on 5 different positions.

\subsection{Wetting evaluations of functionalized fabrics}

The non-wetting properties of the functionalized fabrics were evaluated by the droplets' static contact and rolling on the fabrics according to the previous report (Su et al. 2017; Pan et al. 2018). Besides, the floating experiment of glass glued with functionalized fabrics on the water was also obtained.

\subsection{Antibacterial activity of Ag-MSNs and functionalized fabrics}

The antibacterial activity of Ag-MSNs was determined by the colony count method, and the detailed steps were described in our previous report (Ye et al. 2020). E. coli and S. aureus were cultivated at $37^{\circ} \mathrm{C}$ for $2 \mathrm{~h}$. After cultivation, the bacterial solution ( $E$. coli and $S$. aureus, respectively) were diluted by PSB buffer to $10^{6} \mathrm{CFU} \mathrm{mL}{ }^{-1}$. And then, $1 \mathrm{~mL}$ of the diluted solution was mixed with $1 \mathrm{~mL}$ of PBS buffer containing AgMSNs with a concentration of $40 \mu \mathrm{g} \mathrm{mL}^{-1}$, the newly mixed solution was incubated at a constant temperature and shaking table $\left(37^{\circ} \mathrm{C}, 150 \mathrm{rpm} \mathrm{min}{ }^{-1}\right)$ for $24 \mathrm{~h}$. Finally, $100 \mu \mathrm{L}$ resulting bacterial solution was quickly dispersed onto the LB agar plate by bio-clean SS-Spreader. Bacterial colonies on the cultural plates were counted after incubation at $37^{\circ} \mathrm{C}$ for $24 \mathrm{~h}$. 
To evaluate the antibacterial activity of functionalized fabrics, the fabrics were cut into $1 \mathrm{~cm} \times 2 \mathrm{~cm}$, and three samples were tested in parallel. The functionalized fabrics and the pristine fabrics were all immersed in $5 \mathrm{~mL}$ of bacterial suspension (S. aureus or E. coli, $10^{5} \mathrm{CFU} \mathrm{mL}^{-1}$ ) and subjected to Vortex oscillation for $3 \mathrm{~min}$ to make the bacteria solution contacted with the fabrics well. Then, the samples were taken out and held vertically to allow the droplets remaining on the surface to slide away. Additionally, the samples were transferred into tubes at $37^{\circ} \mathrm{C}$ for $24 \mathrm{~h}$. After the incubation, the fabrics were put into test tubes containing $5 \mathrm{~mL}$ of PBS and experienced a water bath ultrasonication for $10 \mathrm{~min}$, respectively. Finally, $100 \mu \mathrm{L}$ of above-detached bacteria in PBS was spread onto the LB agar plates to incubate at $37^{\circ} \mathrm{C}$ for $24 \mathrm{~h}$. The antibacterial activity of functionalized fabrics was calculated by counting the number of colonies on plates.

To further test the antibacterial activity, inhibition zone test of functionalized fabrics against $S$. aureus, which is regarded as a quantitative technology against $S$. aureus, was conducted according to the AATCC100-2004 standard. As for the inhibition zone test, $100 \mu \mathrm{L}$ of bacterial solution (S. aureus, $10^{6} \mathrm{CFU}$ $\left.\mathrm{mL}^{-1}\right)$ was spread onto LB agar plate, and then the sample $(1.5 \mathrm{~cm} \times 1.5 \mathrm{~cm}$ circles of functionalized fabrics) was put on it to incubate at $37^{\circ} \mathrm{C}$ for $24 \mathrm{~h}$.

\subsection{Mechanical robustness of functionalized fabrics}

We further tested the stability of the antibacterial properties of the fabrics by ultrasonic washing. And the fabrics $(5 \mathrm{~cm} \times 5 \mathrm{~cm})$ were submerged in $50 \mathrm{~mL}$ distilled water for $4 \mathrm{~h}$ under ultrasonication $(40 \mathrm{kHz}, 180$ W). After that, the samples were collected at certain intervals and dried at $105^{\circ} \mathrm{C}$ for $2 \mathrm{~h}$, followed by the measurements of CAs and antibacterial properties, respectively.

Abrasion resistance of fabrics is particularly critical in daily use. Herein, the wear resistance of functionalized fabrics was tested by sandpaper abrasion. A $200 \mathrm{~g}$ weight was put on the fabric while it was placed facedown to the sandpaper (standard sandpaper, grit no. 360). Then, the fabric was moved back and forth at about $2 \mathrm{~cm} / \mathrm{s}$. Each iteration was counted as a cycle, and CAs and antibacterial properties of the fabric were tested after every 100 abrasion cycles.

\section{Results And Discussion}

\subsection{Characterization of Ag-MSNs and F-MSNs}

The morphologies of the as-prepared Ag-MSNs and F-MSNs were shown in Fig. 1. As shown in Fig. 1a, the black spots were silver particles, which were bonded with MSNs, and the synthetic Ag-MSNs presented a ball-and-stick structure (Fig.1d). The sphericity of F-MSNs was irregular, with some roughness (Fig. $1 \mathrm{~b}$ ), and the particle size of F-MSNs was about 50-60 nm (Fig.1f). The stretching modes of the Si-O-Si groups belonged to MSNs and F-MSNs were shown at $804 \mathrm{~cm}^{-1}$ and $463 \mathrm{~cm}^{-1}$, while the hydroxyl stretching of Si-OH groups was shown at $3372 \mathrm{~cm}^{-1}$. As for F-MSNs, the absorption peaks at $1147 \mathrm{~cm}^{-1}$ and $1207 \mathrm{~cm}^{-1}$ could be ascribed to antisymmetric and symmetric C-F stretching modes (Fig. 
1c). Additionally, the element mapping results of two types of nanoparticles in Fig. 1e showed Ag-MSNs contained three elements: $\mathrm{Si}, \mathrm{O}$, and $\mathrm{Ag}$, while F-MSNs contained $\mathrm{Si}$, O, and $\mathrm{F}$ elements (Fig. 1g). These results indicated the Ag-MSNs and F-MSNs were successfully synthetic.

\subsection{Characterizations of functionalized fabrics}

The optical photo of THF solution mixed with two functionalized nanoparticles after 15 min sonication was shown in Fig. 2a. The solution was black resulting from the presence of Ag-MSNs. As shown in Fig. $2 \mathrm{~b}$, the surface of functionalized fabrics was covered with nanoparticles, and four elements: $\mathrm{Si}, \mathrm{O}, \mathrm{Ag}$, and $F$ were well shown in the element mapping. Due to the nanostructure of the two kinds of nanoparticles and the microstructure of the fabrics surface, combined with the hydrophobic properties of PDMS and FMSNs, the functionalized fabrics had excellent superhydrophobicity $\left(C A=151^{\circ}, S A=6^{\circ}\right)$ shown in Fig. 2c. To further evaluate the superhydrophobicity of functionalized fabrics, Fig. $2 \mathrm{~d}$ and e showed the photos of a water droplet touching and leaving the pristine fabrics and functionalized fabrics, respectively. The droplet was forced to contact the fabrics surface sufficiently with an obvious change in sphericity, and when it was lifted lightly, the water droplet showed almost no deformation in Fig. 2e, while the water droplet was quickly immersing in the pristine fabrics in Fig. 2d, which confirming the extremely low water adhesion for functionalized fabrics (Gu et al. 2019).

\subsection{Non-wetting properties of the functionalized fabrics}

The superhydrophobicity of the functionalized fabrics can reduce the adhesion of bacteria in the aqueous solution (Zhu et al. 2020). To intuitively reflect the superhydrophobicity of functionalized fabrics, three related experiments were designed as follows. Through the static and sliding experiments of droplets, we further verified that the fabrics had excellent superhydrophobicity. As shown in Fig. 3a left and Video. S1, five kinds of common water-based droplets (milk, cola, coffee, juice, water) were dropped on the functionalized fabrics (bottom) and the pristine fabric (top), respectively. Compared with the pristine fabrics in which the droplets permeated rapidly, the functionalized fabrics possessed well non-wetting properties that the droplets remained spherical. In addition, the droplets would be easily absorbed away by the paper and there were no droplets left on the surface of the functionalized fabrics (Fig. 3a right and Video. S2). After that, we glued the functionalized fabrics and the pristine fabrics to the glass sheet with double-sided adhesive, respectively. As shown in Fig. 3b and Video. S3, when they were placed in the dyed water at the same time, we found that the functionalized fabrics with the glass plate could float on the water surface, while the pristine fabrics with the glass plate would sink in the water, which further proved that the functionalized fabrics had excellent hydrophobicity. As shown in Fig. 3c and Video. S4, the droplets could slide quickly from the top to the bottom of the functionalized fabrics. The experiment of droplets' roll-off further confirmed the non-stick performance of the functionalized fabrics.

\subsection{Antibacterial properties of Ag-MSNs and the functionalized fabrics}

The antibacterial ability of Ag-MSNs is a key factor in determining the functionalized fabrics, and we quantitatively obtained the antibacterial properties of Ag-MSNs. As shown in Fig. S1, when the 
concentration of Ag-MSNs reached $20 \mu \mathrm{g} \mathrm{mL}^{-1}$, the antibacterial rates for $E$. coli and $S$. aureus were both as high as $99.9 \%$. As well known, long-term antibacterial properties of the functionalized fabrics play an important role in daily use, and the stability of the antibacterial properties can provide reliable protection (Lu et al. 2015). As shown in Fig. 4a, functionalized fabrics, named TF, had excellent antibacterial properties compared with the pristine fabrics, named F. In addition, the long-term antibacterial performance of functionalized fabrics was verified through the colony counting method. With time going by, the antibacterial rates of the functionalized fabrics decreased gradually due to the continuous release of $\mathrm{Ag}^{+}$(Fig. 4b). Thanks to the synergistic effect of superhydrophobic antifouling and Ag-MSNs antibacterial properties, the antibacterial rates of the functionalized fabrics could still reach $92.3 \%$ ( $E$. coli) and $90.1 \%$ (S. aureus) even after $30 \mathrm{~d}$, which was sufficient to ensure that the functionalized fabrics had stable protective effects during daily use.

Antibacterial properties of the functionalized fabrics were more directly reflected in Fig. 5. As-prepared fabrics could keep an excellent zone of inhibition for $25 \mathrm{~d}$ because of the continuous release of $\mathrm{Ag}^{+}$from the Ag-MSNs coated on the surface of the fabrics (Nie et al. 2018). The diameters of the inhibition zones were well observed, which intuitively revealed that the as-prepared fabrics possessed prominent antibacterial properties. In addition, we further explored the influence of the concentration of PDMS in the dip-coating solution on the zone of inhibition and found that when the concentration of PDMS reached $0.02 \mathrm{~g} \mathrm{~mL}^{-1}$, the zone of inhibition did not appear (Fig. S2a). The long-term antibacterial properties of asprepared fabrics might result from the PDMS which slowed down the release of $\mathrm{Ag}^{+}$.

\subsection{Mechanical robustness of the functionalized fabrics}

Excellent mechanical robustness is a prerequisite for the reliable protection of functionalized fabrics. Ultrasonic washing and sandpaper abrasion tests were used to evaluate the robustness of the fabrics, and shown in Fig. 6. The changes of morphology were observed by SEM in Fig. 6a and c. After ultrasonic washing for $150 \mathrm{~min}$, there were still many functionalized nanoparticles on the surface of the fabrics (Fig. 6a). Besides, the CAs and antibacterial rates (S. aureus) of the functionalized fabrics could still reach $144^{\circ}$ and $94.3 \%$, which meant the fabrics were extremely stable to keep their hydrophobicity and antibacterial properties. During the daily use of cotton fabrics, wear occurs over time. The wear resistance of functionalized fabrics was investigated through a mechanical wear test by using sandpaper abrasion. As shown in Fig. 6c, the texture structure of the surface of fabrics was greatly destroyed after 500 times abrasion. Interestingly, the CAs and the antibacterial rate (S. aureus) only decreased to $140^{\circ}$ and $90.2 \%$, which proved that the fabrics still had excellent antifouling and antibacterial properties. The reason why the worn fabric still had good antibacterial properties was that the functionalized nanoparticles were firmly bonded to the surface of the fabric fibers by PDMS. Even if the surface fibers were damaged, the silver nanoparticles on the remaining fibers could continue releasing $\mathrm{Ag}^{+}$to achieve the sterilization effect.

\section{Conclusion}


In this study, two kinds of functionalized nanoparticles, Ag-MSNs and F-MSNs, were well synthesized. Two kinds of functionalized nanoparticles, together with PDMS, were added into the THF solution to prepare a functionalized coating solution, which was coated onto the cotton fabrics through the dipcoating method. Furthermore, the functionalized fabrics with both long-term antibacterial effect and superhydrophobic antifouling function were successfully tested. Experimental studies showed that the prepared fabrics had effective long-term inhibition effects on $S$. aureus, and the inhibition effects could reach more than $25 \mathrm{~d}$. Besides, the superhydrophobic properties resulted from the existence of F-MSNs, which makes the functionalized fabrics inhibit the anti-adhesion of bacteria. It is because of the synergy of antibacterial properties and antiadhesion of bacteria, the antibacterial rates of functionalized fabrics up to $92.3 \%$ (E. coli) and $90.1 \%$ (S. aureus) even after $30 \mathrm{~d}$. More significantly, considering the simple preparation process, low cost, antifouling, and long-term antibacterial properties of as-prepared fabrics, these functionalized fabrics will have great application prospects in medical materials and special textiles in the future.

\section{Declarations}

\section{Funding}

This study was funded by [National Natural Science Foundation of China (31671021) and Natural Science Foundation of Tianjin City (19JCYBJC17200)]

\section{Conflicts of interests}

All authors certify that they have no affiliation with or involvement in any organization or entity with any financial interest or non-financial interest in the subject matter or materials discussed in this manuscript.

\section{Availability of data and material}

All authors make sure that all data and materials support the published claims and comply with field standards.

\section{Code availability}

All authors make sure that software applications or custom codes support the published claims and comply with field standards.

\section{Authors' contributions}

All authors contributed to the study conception and design. Material preparation, data collection, and analysis were performed by [Ye Zhanpeng], [Rong Hui], and [Li Shuangyang]. The first draft of the manuscript was written by [Ye Zhanpeng] and all authors commented on previous versions of the manuscript. All authors read and approved the final manuscript.

\section{Acknowledgments}


We gratefully acknowledge the financial support provided by National Natural Science Foundation of China (31671021) and Natural Science Foundation of Tianjin City (19JCYBJC17200).

\section{References}

1. Xu Q, Xie L, Diao H, Li F, Zhang Y, Fu F, Liu X (2017) Antibacterial cotton fabric with enhanced durability prepared using silver nanoparticles and carboxymethyl chitosan. Carbohydr. Polym. 177: 187-193. https://doi.org/10.1016/j.carbpol.2017.08.129

2. Wu M, Ma B, Pan T, Chen S, Sun J (2016) Silver-Nanoparticle-Colored Cotton Fabrics with Tunable Colors and Durable Antibacterial and Self-Healing Superhydrophobic Properties. Adv. Funct. Mater. 26: 569-576. https://doi.org/10.1002/adfm.201504197.

3. Elshaarawy RFM, Seif GA, El-Naggar ME, Mostafa TB, El-Sawi EA (2019) In-situ and ex-situ synthesis of poly-(imidazolium vanillyl)-grafted chitosan/silver nanobiocomposites for safe antibacterial finishing of cotton fabrics. Eur. Polym. J. 116: 210-221. https://doi.org/10.1016/j.eurpolymj.2019.04.013.

4. Zhang LJ, Guerrero-Juarez CF, Hata T, Bapat SP, Ramos R, Plikus MV, Gallo RL (2015) Innate immunity. Dermal adipocytes protect against invasive Staphylococcus aureus skin infection. Science 347: 67-71. https://doi.org/10.1126/science.1260972.

5. Li P, Poon YF, Li W, Zhu HY, Yeap SH, Cao Y, Qi X, Zhou C, Lamrani M, Beuerman RW, Kang ET, Mu Y, Li CM, Chang MW, Leong SS, Chan-Park MB (2011) A polycationic antimicrobial and biocompatible hydrogel with microbe membrane suctioning ability. Nat. Mater. 10:149-156. https://doi.org/10.1038/nmat2915.

6. Liu T, Yin B, He T, Guo N, Dong L, Yin Y (2012) Complementary effects of nanosilver and superhydrophobic coatings on the prevention of marine bacterial adhesion. ACS Appl. Mater. Interfaces 4: 4683-4690. https://doi.org/10.1021/am301049v.

7. Holt BA, Gregory SA, Sulchek T, Yee S, Losego MD (2018) Aqueous Zinc Compounds as Residual Antimicrobial Agents for Textiles. ACS Appl. Mater. Interfaces 10: 7709-7716. https://doi.org/10.1021/acsami.7b15871.

8. Rizzello L, Pompa PP (2014) Nanosilver-based antibacterial drugs and devices: mechanisms, methodological drawbacks, and guidelines. Chem. Soc. Rev. 43: 1501-1518. https://doi.org/10.1039/c3cs60218d.

9. Lin J, Chen X, Chen C, Hu J, Zhou C, Cai X, Wang W, Zheng C, Zhang P, Cheng J, Guo Z, Liu H (2018) Durably Antibacterial and Bacterially Antiadhesive Cotton Fabrics Coated by Cationic Fluorinated Polymers. ACS Appl. Mater. Interfaces 10: 6124-6136. https://doi.org/10.1021/acsami.7b16235.

10. Fan XL, Hu M, Qin ZH, Wang J, Chen XC, Lei WX, Ye WY, Jin Q, Ren KF, Ji J (2018) Bactericidal and Hemocompatible Coating via the Mixed-Charged Copolymer. ACS Appl. Mater. Interfaces 10: 1042810436. https://doi.org/10.1021/acsami.7b18889. 
11. Fu Y, Jiang J, Zhang Q, Zhan X, Chen F (2017) Robust liquid-repellent coatings based on polymer nanoparticles with excellent self-cleaning and antibacterial performances. J. Mater. Chem. A 5: 275284. https://doi.org/10.1039/c6ta06481g.

12. Jin C, Liu X, Tan L, Cui Z, Yang X, Zheng Y, Yeung KWK, Chu PK, Wu S (2018) Ag/AgBr-loaded mesoporous silica for rapid sterilization and promotion of wound healing. Biomater. Sci. 6: 17351744. https://doi.org/10.1039/c8bm00353j.

13. Ouadil B, Amadine O, Essamlali Y, Cherkaoui O, Zahouily M (2019) A new route for the preparation of hydrophobic and antibacterial textiles fabrics using Ag-loaded graphene nanocomposite. Colloids Surf. Physicochem. Eng. Aspects 579: 123713. https://doi.org/10.1016/j.colsurfa.2019.123713.

14. Syafiuddin A (2019) Toward a comprehensive understanding of textiles functionalized with silver nanoparticles. J. Chin. Chem. Soc. 66: 793-814. https://doi.org/10.1002/jccs.201800474.

15. Krishnan PD, Banas D, Durai RD, Kabanov D, Hosnedlova B, Kepinska M, Fernandez C, RuttkayNedecky B, Nguyen HV, Farid A, Sochor J, Narayanan VHB, Kizek R (2020) Silver Nanomaterials for Wound Dressing Applications. Pharmaceutics 12:12090821.

https://doi.org/10.3390/pharmaceutics12090821.

16. Yu W, Li X, He J, Chen Y, Qi L, Yuan P, Ou K, Liu F, Zhou Y, Qin X (2021) Graphene oxide-silver nanocomposites embedded nanofiber core-spun yarns for durable antibacterial textiles. J. Colloid Interface Sci. 584: 164-173. https://doi.org/10.1016/j.jcis.2020.09.092.

17. Zhang S, Tang Y, Vlahovic B (2016) A Review on Preparation and Applications of Silver-Containing Nanofibers. Nanoscale Res. Lett. 11: 80. https://doi.org/10.1186/s11671-016-1286-z.

18. Xu Q, Li R, Shen L, Xu W, Wang J, Jiang Q, Zhang L, Fu F, Fu Y, Liu X (2019) Enhancing the surface affinity with silver nano-particles for antibacterial cotton fabric by coating carboxymethyl chitosan and l-cysteine. Appl. Surf. Sci. 497:143673. https://doi.org/10.1016/j.apsusc.2019.143673.

19. Chauhan P, Kumar A, Bhushan B (2019) Self-cleaning, stain-resistant and anti-bacterial superhydrophobic cotton fabric prepared by simple immersion technique. J. Colloid Interface Sci. 535: 66-74. https://doi.org/10.1016/j.jcis.2018.09.087.

20. Ou J, Wu B, Xue M, Wang F (2019) Silver ions anchored to fabric via coordination: Evaluation on washing durability and antibacterial activity. Mater. Lett. 237: 134-136. https://doi.org/10.1016/j.matlet.2018.11.090.

21. Qiu Q, Chen S, Li Y, Yang Y, Zhang H, Quan Z, Qin X, Wang R, Yu J (2020) Functional nanofibers embedded into textiles for durable antibacterial properties. Chem. Eng. J. 384:123241. https://doi.org/10.1016/j.cej.2019.123241.

22. Kim J-H, Mirzaei A, Kim HW, Kim SS (2018) Novel superamphiphobic surfaces based on micro-nano hierarchical fluorinated $\mathrm{Ag} / \mathrm{SiO}_{2}$ structures. Appl. Surf. Sci. 445: 262-271. https://doi.org/10.1016/j.apsusc.2018.03.148.

23. Li S, Zhu T, Huang J, Guo Q, Chen G, Lai Y (2017) Durable antibacterial and UV-protective Ag/TiO ${ }_{2} @$ fabrics for sustainable biomedical application. Int. J. Nanomed. 12: 2593-2606. https://doi.org/10.2147/IJN.S132035. 
24. Wang D, Sun Q, Hokkanen MJ, Zhang C, Lin FY, Liu Q, Zhu SP, Zhou T, Chang Q, He B, Zhou Q, Chen L, Wang Z, Ras RHA, Deng X (2020) Design of robust superhydrophobic surfaces. Nature 582: 55-59. https://doi.org/10.1038/s41586-020-2331-8.

25. Li S, Huang J, Chen Z, Chen G, Lai Y (2017) A review on special wettability textiles: theoretical models, fabrication technologies and multifunctional applications. J. Mater. Chem. A 5: 31-55. https://doi.org/10.1039/c6ta07984a.

26. Nie W, Dai X, Li D, McCoul D, Gillispie GJ, Zhang Y, Yu B, He C (2018) One-Pot Synthesis of Silver Nanoparticle Incorporated Mesoporous Silica Granules for Hemorrhage Control and Antibacterial Treatment. ACS Biomater. Sci. Eng. 4: 3588-3599. https://doi.org/10.1021/acsbiomaterials.8b00527.

27. Wu D, Shi X, Zhao F, Chilengue STF, Deng L, Dong A, Kong D, Wang W, Zhang J (2019) An injectable and tumor-specific responsive hydrogel with tissue-adhesive and nanomedicine-releasing abilities for precise locoregional chemotherapy. Acta Biomater. 96: 123-136. https://doi.org/10.1016/j.actbio.2019.06.033.

28. Su X, Li H, Lai X, Zhang L, Wang J, Liao X, Zeng X (2017) Vapor-Liquid Sol-Gel Approach to Fabricating Highly Durable and Robust Superhydrophobic Polydimethylsiloxane@Silica Surface on Polyester Textile for Oil-Water Separation. ACS Appl. Mater. Interfaces 9: 28089-28099. https://doi.org/10.1021/acsami.7b08920.

29. Pan S, Guo R, Bjornmalm M, Richardson JJ, Li L, Peng C, Bertleff-Zieschang N, Xu W, Jiang J, Caruso F (2018) Coatings super-repellent to ultralow surface tension liquids. Nat. Mater. 17: 1040-1047. https://doi.org/10.1038/s41563-018-0178-2.

30. Ye Z, Li S, Zhao S, Deng L, Zhang J, Dong A (2021) Textile coatings configured by doublenanoparticles to optimally couple superhydrophobic and antibacterial properties. Chem. Eng. J. 420:127680. https://doi.org/10.1016/j.cej.2020.127680.

31. Gu Z, Kothary P, Sun CH, Gari A, Zhang Y, Taylor C, Jiang P (2019) Evaporation-Induced Hierarchical Assembly of Rigid Silicon Nanopillars Fabricated by a Scalable Two-Level Colloidal Lithography Approach. ACS Appl. Mater. Interfaces 11: 40461-40469. https://doi.org/10.1021/acsami.9b12388.

32. Zhu R, Liu M, Hou Y, Zhang L, Li M, Wang D, Fu S (2020) One-Pot Preparation of Fluorine-Free Magnetic Superhydrophobic Particles for Controllable Liquid Marbles and Robust Multifunctional Coatings. ACS Appl. Mater. Interfaces 12: 17004-17017. https://doi.org/10.1021/acsami.9b22268.

33. Lu Z, Xiao J, Wang Y, Meng M (2015) In situ synthesis of silver nanoparticles uniformly distributed on polydopamine-coated silk fibers for antibacterial application. J. Colloid Interface Sci. 452: 8-14. https://doi.org/10.1016/j.jcis.2015.04.015.

\section{Figures}




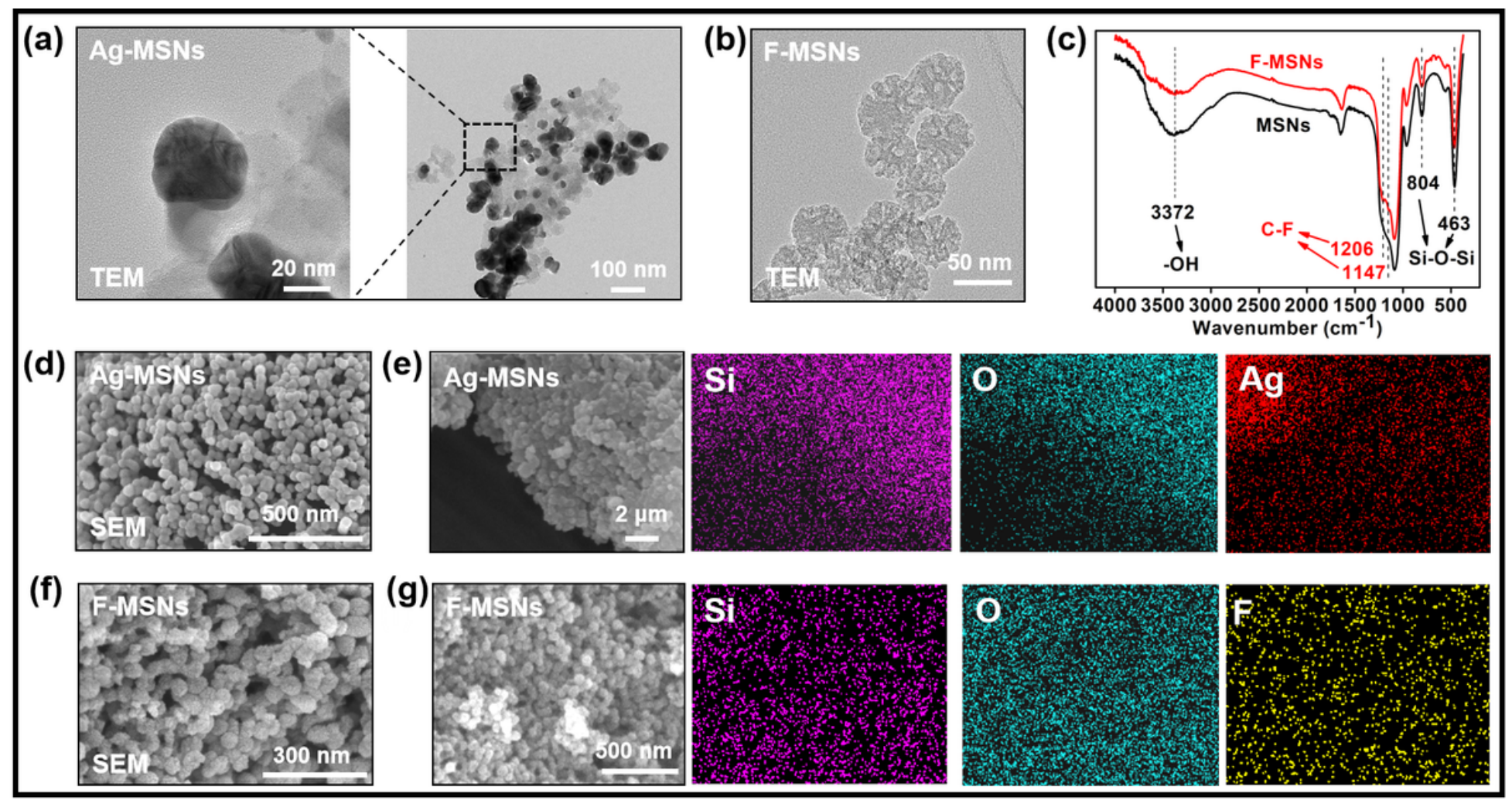

Figure 1

Characterizations of functionalized nanoparticles. (a, b) TEM images of two nanoparticles (Ag-MSNs and F-MSNs). (c) FTIR spectra of MSNs and F-MSNs. (d, f) SEM images of Ag-MSNs and F-MSNs. (e, g) Element mapping images of Ag-MSNs and F-MSNs.

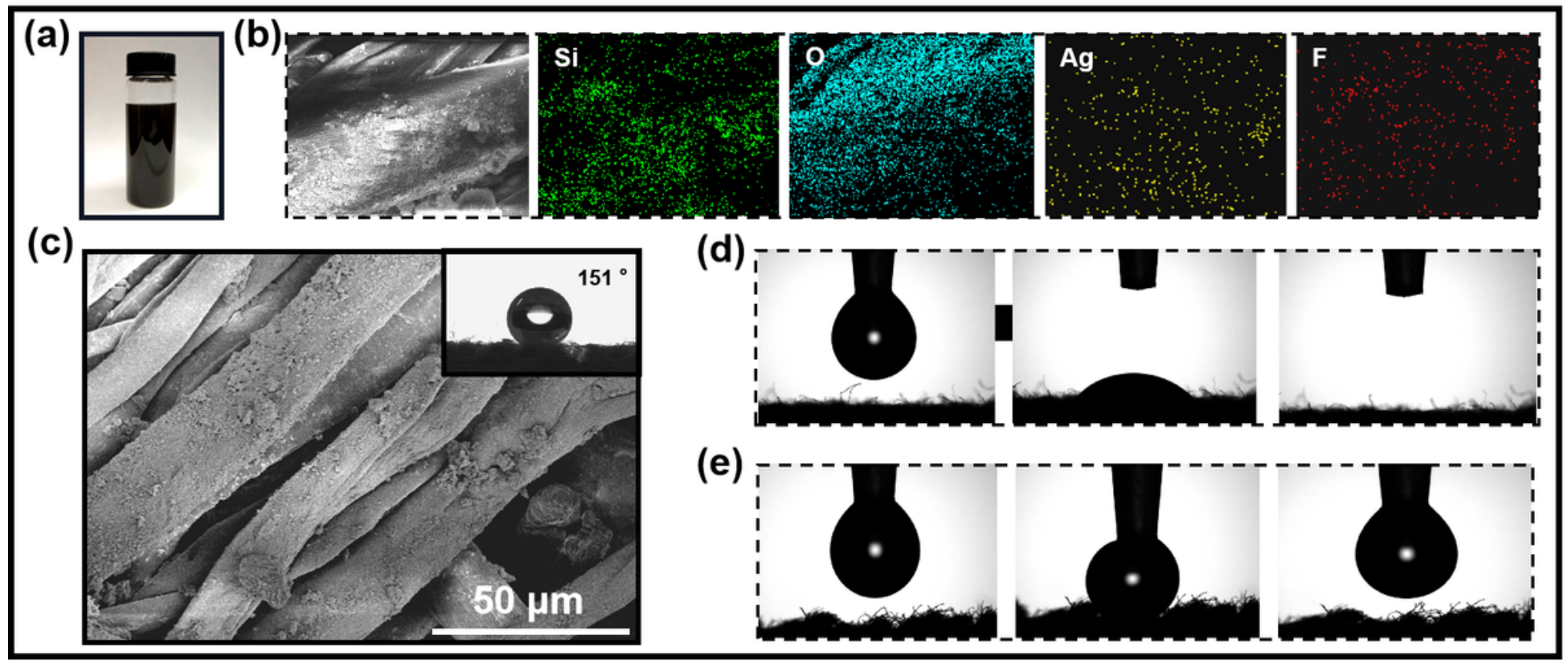

Figure 2

Characterizations of functionalized fabrics. (a) Disperse solution with functional nanoparticles. (b) The element mapping images of functionalized fabrics. (c) The SEM and CAs images of functionalized 
fabrics. Droplet wetting rates on pristine fabrics (d) and functionalized fabrics (e).

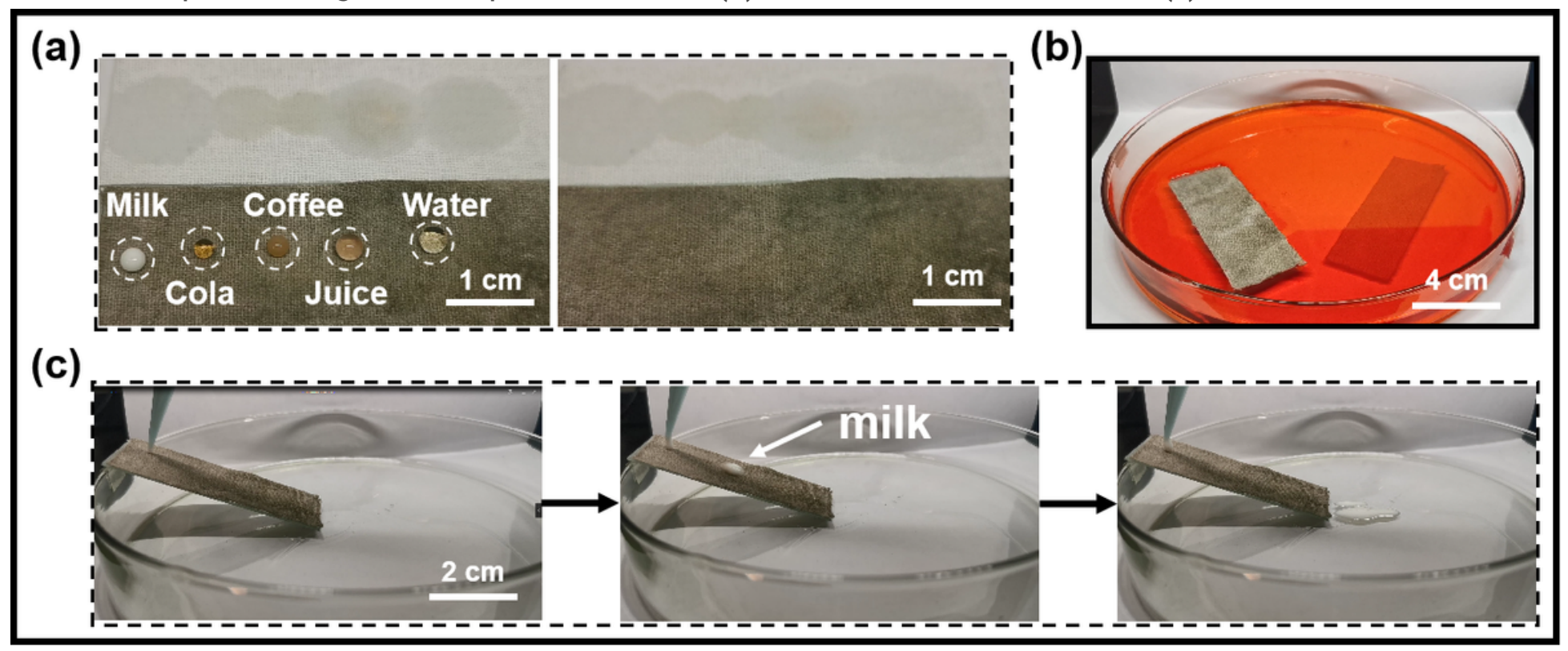

Figure 3

Non-wetting properties of functionalized fabrics. (a) The still images of different droplets on functionalized fabrics (bottom) and pristine fabrics (top). (b) the glass which was glued with functionalized fabrics (left) and pristine fabrics (right). (c) The sliding images of milk droplets on functionalized fabrics.

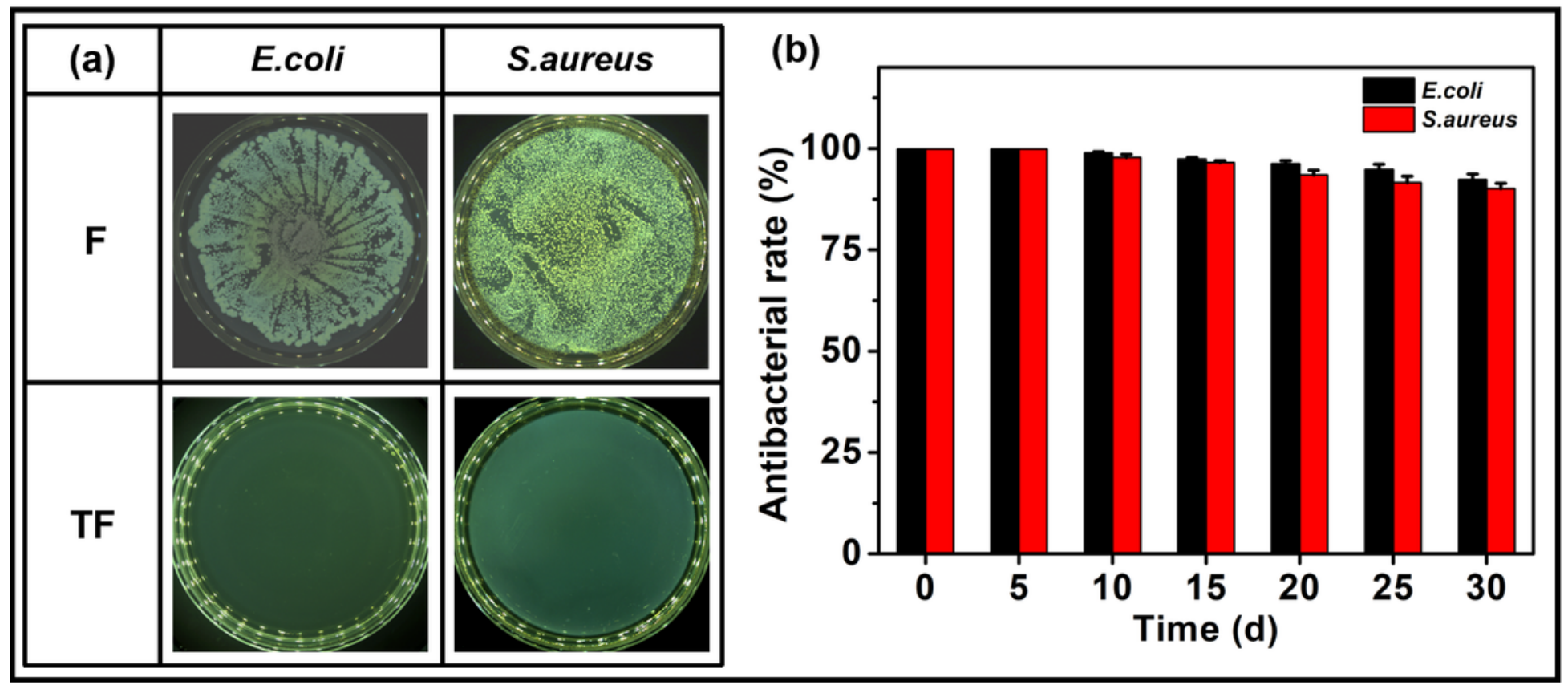

Figure 4

Antibacterial properties of functionalized fabrics. (a) The growth of E. coli and S. aureus on the agar plate for different fabrics. (b) Antibacterial rates of functionalized fabrics from the first day to the 30th day. 


\section{S.aureus}

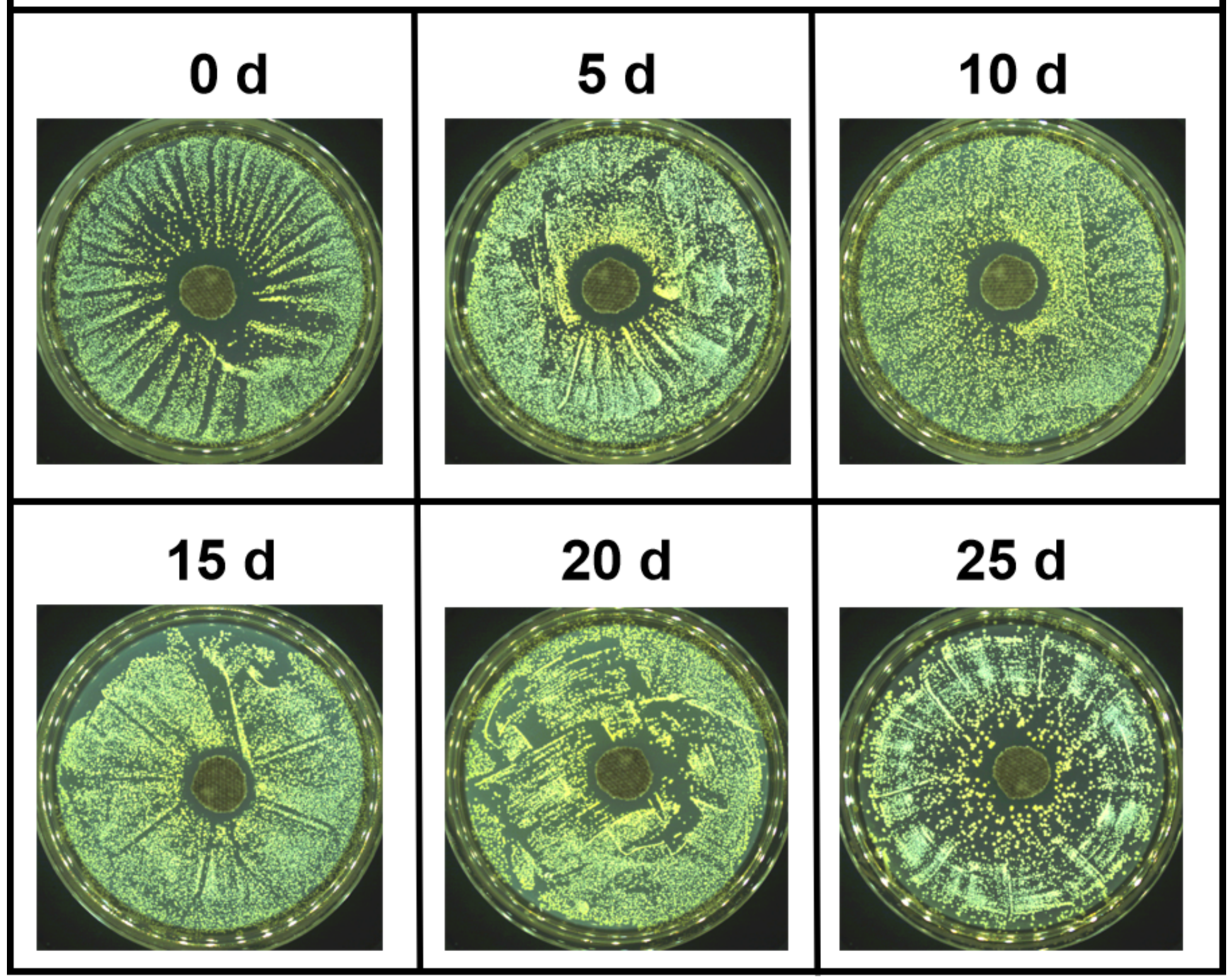

Figure 5

Zone of inhibition for long-term antibacterial properties of functionalized fabrics. 


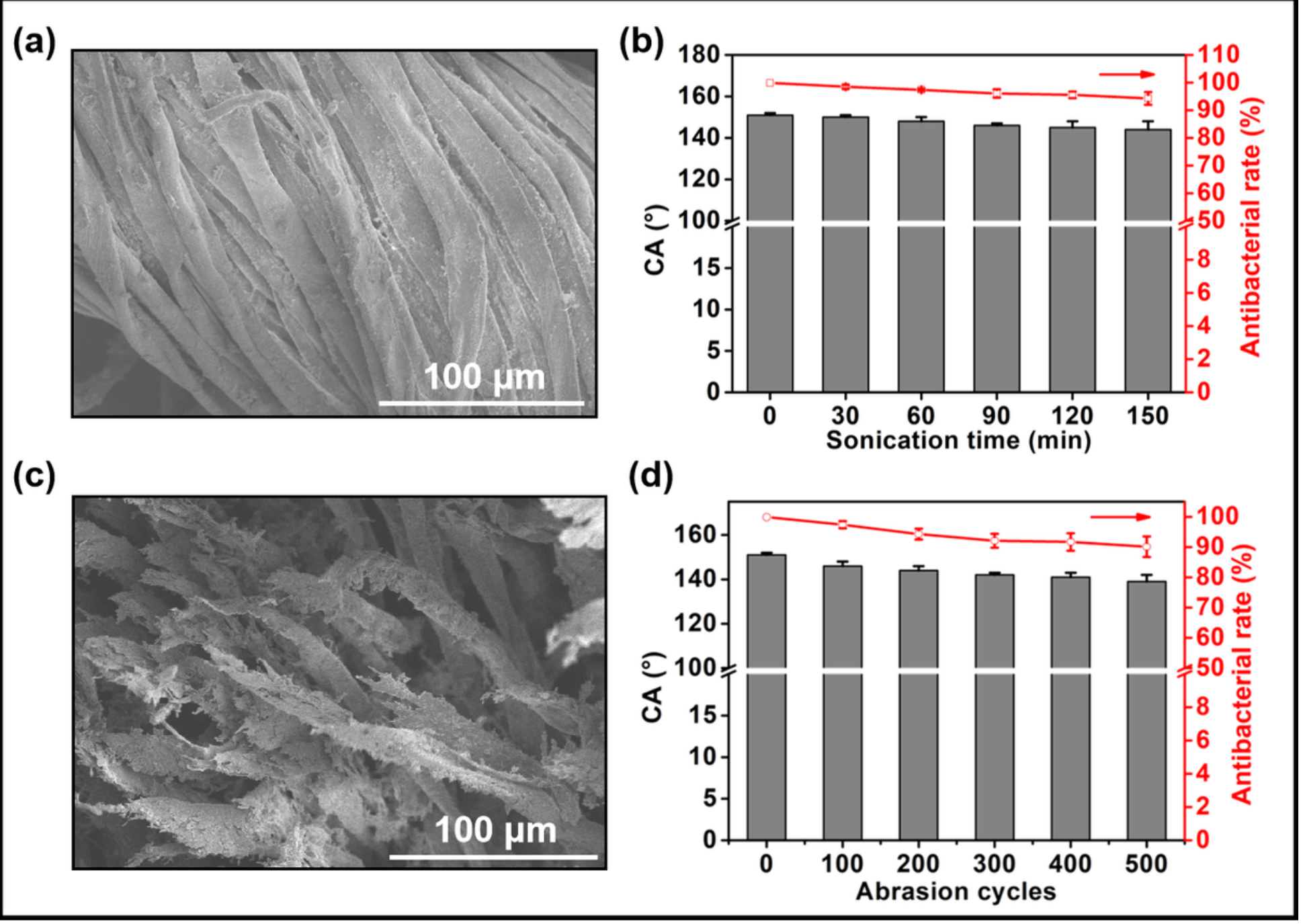

Figure 6

Mechanical robustness of functionalized fabrics. The SEM images of functionalized fabrics after 150 min ultrasonic washing (a) and 500 abrasion cycles (c). The CAs and antibacterial rates of functionalized fabrics after sonication (b) and abrasion (d).

\section{Supplementary Files}

This is a list of supplementary files associated with this preprint. Click to download.

- Figure.S1.tif

- Figure.S2.tif

- Scheme.tif

- Supportinginformation.docx

- Video.S1.mp4

- Video.S2.mp4 
- Video.S3.mp4

- Video.S4.mp4 